\title{
Large Identified Pyramidal Cells in Macaque Motor and Premotor Cortex Exhibit “Thin Spikes”: Implications for Cell Type Classification
}

\author{
Ganesh Vigneswaran, ${ }^{\star}$ Alexander Kraskov, ${ }^{\star}$ and Roger N. Lemon \\ Sobell Department of Motor Neuroscience and Movement Disorders, UCL Institute of Neurology, London WC1N 3BG, United Kingdom
}

Recent studies have suggested that extracellular recordings of putative cortical interneurons have briefer spikes than those of pyramidal neurons, providing a means of identifying cortical cell types in recordings from awake monkeys. To test this, we investigated the spike duration of antidromically identified pyramidal tract neurons (PTNs) recorded from primary motor (M1) or ventral premotor cortex (area F5) in 4 awake macaque monkeys. M1 antidromic latencies (ADLs) were skewed toward short ADLs (151 PTNs; 0.5-5.5 ms, median $1.1 \mathrm{~ms}$ ) and significantly different from that of F5 ADLs (54 PTNs; $1.0-6.9 \mathrm{~ms}$, median $2.6 \mathrm{~ms}$ ). The duration of PTN spikes, recorded with a high-pass filter of $300 \mathrm{~Hz}$ and measured from the negative trough to the positive peak of the spike waveform, ranged from 0.15 to $0.71 \mathrm{~ms}$. Importantly, we found a positive linear correlation between ADL and spike duration in both $\mathrm{M} 1\left(R^{2}=0.40, p<0.001\right)$ and F5 $\left(R^{2}=0.57\right.$, $p<0.001$ ). Thus PTNs with the shortest ADL (fastest axons) had the briefest spikes, and since PTN soma size is correlated with axon size and conduction velocity, it is likely that the largest pyramidal neurons (Betz cells in M1) have spikes with short durations $(0.15-0.45 \mathrm{~ms})$, which overlap heavily with those reported for putative interneurons in previous studies in non-primates. In summary, one class of physiologically identified cortical pyramidal neuron exhibits a wide variety of spike durations and the results suggest that spike duration alone may not be a reliable indicator of cell type.

\section{Introduction}

It is becoming increasingly important to determine the identity of cortical neurons involved in a wide range of different brain functions. The neocortex is comprised of a range of different pyramidal cells and interneurons, and distinguishing between these two groups of neurons in recordings made from awake, behaving animals is a key issue. Early investigators first suggested that interneurons, with high spontaneous firing rates, had "thin" action potentials of short duration and could be distinguished from pyramidal cells with longer action potentials and lower, regular spiking pattern of discharge (Mountcastle et al., 1969). These differences were subsequently confirmed by detailed intracellular studies in brain slices from rodents (Connors et al., 1982; McCormick et al., 1985; Contreras, 2004).

In vivo studies using high-density recordings from rat neocortex (Barthó et al., 2004) further suggested that the trough-to-peak duration of the unfiltered extracellular spike waveform, which is directly related to intracellular spike width at half-amplitude

\footnotetext{
Received June 21, 2011; revised July 22, 2011; accepted Aug. 17, 2011.

Author contributions: G.V., A.K., and R.N.L. designed research; G.V., A.K., and R.N.L. performed research; G.V. and A.K. analyzed data; G.V., A.K., and R.N.L. wrote the paper.

This work was supported by the University College London (UCL) Grand Challenge Scheme, the Wellcome Trust, and the UK National Centre for the 3Rs. We thank Stuart Baker and Claire Witham for providing us with part of the data, and Samantha Shepherd for technical assistance.

${ }^{*} G$.V. and A.K. contributed equally to the work presented here.

Correspondence should be addressed to: Dr. Roger N. Lemon, Sobell Department of Motor Neuroscience and Movement Disorders, UCL Institute of Neurology, Queen Square, London WC1N 3BG, UK. E-mail: r.lemon@ucl.ac.uk.

DOI:10.1523/JNEUROSCI.3142-11.2011

Copyright $\odot 2011$ the authors $\quad 0270-6474 / 11 / 3114235-08 \$ 15.00 / 0$
}

(Henze et al., 2000; Gold et al., 2006), provided the most reliable means of distinguishing recordings from putative inhibitory interneurons vs pyramidal neurons. A number of recent reports in the awake, behaving monkey have applied this criterion as a means of identifying different cell types in cortical recordings, allowing better definition of local cortical circuitry underlying a variety of brain mechanisms involved in motor planning (Kaufman et al., 2010), control of arm direction (Merchant et al., 2008) and attention (Mitchell et al., 2007).

To verify the hypothesis that spike durations of extracellular action potentials can be used as a reliable classifier of cell type in the awake monkey, recordings from identified interneurons and pyramidal cells are needed. Pyramidal tract neurons (PTNs) are one class of layer $\mathrm{V}$ pyramidal neuron found in cortical motor areas which can be unambiguously identified using pyramidal tract stimulation (Evarts, 1964; Lemon, 1984). Most PTNs have axons which reach the spinal cord in the corticospinal tract (Humphrey and Corrie, 1978).

Intracellular studies in the cat have shown a clear relationship between the duration of the action potential and axonal conduction velocity (Calvin and Sypert, 1976; Sakai and Woody, 1988; Baranyi et al., 1993), with "slow" PTNs having longer spikes than "fast" PTNs. There is, however, a paucity of comparable data on extracellular PTN spike duration from the awake, behaving monkey, in which the conduction velocity and organization of the corticospinal tract are different from the cat (Lemon, 2008). As a result, it remains unclear whether or not spike duration could be reliably used to distinguish between interneurons and all types of pyramidal neurons in extracellular recordings in awake monkeys. 
In this study we made extracellular recordings from physiologically identified PTNs in the awake macaque primary motor (M1) and ventral premotor cortex (area F5) and analyzed the distribution of their spike durations. We were particularly focused on measuring the spike durations of fast PTNs to see whether these values overlapped with those claimed for putative interneurons in the awake primate. We also made comparisons of PTN spikes with those of other unidentified neurons in the same recordings, and also with mean values of spike durations of interneurons and pyramidal cells reported in the literature.

\section{Materials and Methods}

Experiments were performed on two adult purpose-bred rhesus (Macaca mulatta) monkeys (M43, female $5.5 \mathrm{~kg}$ and M44, male $7.1 \mathrm{~kg}$ ). Additional recorded data from two other purpose-bred rhesus monkeys ( $M$, female 6.0 $\mathrm{kg}$ and L, female $5.3 \mathrm{~kg}$ ) were kindly provided by Prof. Stuart Baker's laboratory at Newcastle University (Witham and Baker, 2007). All experimental procedures were approved by the respective Local Ethical Procedures committees and performed in accordance with the UK Animals (Scientific Procedures) Act.

Recordings were made during performance of skilled grasping tasks with the contralateral hand. A full description of the tasks and of the surgical procedures used to prepare the monkeys for recording has been published previously (Witham and Baker, 2007; Kraskov et al., 2009). All monkeys were chronically implanted with a pair of fine tungsten stimulating electrodes in the medullary pyramid for subsequent antidromic identification of PTNs. These electrodes were confirmed to be located in the ipsilateral pyramidal tract by a number of electrophysiological and histological tests (Olivier et al., 2001; Kraskov et al., 2009).

Cortical recordings. Neurons were recorded in the hand areas of primary motor cortex M1 (in all 4 monkeys) and ventral premotor cortex (area F5) in two of them (M43 and M44). It is known that a large proportion of the macaque corticospinal tract is derived from the cortex outside M1 (Dum and Strick, 1991), and that corticospinal neurons in secondary motor areas are generally smaller and have slower conduction velocities than those in M1 (Murray and Coulter, 1981; Macpherson et al., 1982; Maier et al., 2002; Kraskov et al., 2009). Therefore comparison of spike durations for PTNs recorded in M1 vs those in premotor cortex (area F5) was of particular importance. Recordings were made with the Thomas Recording multiple microelectrode system, using quartz glass insulated platinum-iridium microelectrodes with impedances of $\sim 1-2$ $\mathrm{M} \Omega$. Neuronal data were preamplified $(20 \times)$ using a standard Thomas headstage, further amplified (giving a final gain of 20,000, typically) and analog filtered using custom built amplifiers. Second-order high-pass filter with $300 \mathrm{~Hz}$ cutoff frequency and first-order low-pass filter at $6 \mathrm{kHz}$ were used; some data were recorded using wide band filter settings $(10 \mathrm{~Hz}-6$ $\mathrm{kHz}$ ). Spike data were digitized using a sampling frequency of $25 \mathrm{kHz}$.

PTNidentification. Details of PTN identification used are given in Kraskov et al. (2009). In brief, we searched for PTNs by looking for a latency-invariant antidromic response to stimulation of the pyramidal tract (PT) with single shocks of a $250-300 \mu \mathrm{A}$ (biphasic pulse, each phase $0.2 \mathrm{~ms}$ ). Once a PTN spike was clearly present in the recording, we determined its antidromic latency (ADL). This was measured from the beginning of the stimulus artifact to the first inflection in the antidromic spike (Fig. 1; see example in inset). We then discriminated the spontaneous spikes originating from the PTN on-line and performed a collision test (Lemon, 1984; Baker et al., 1999). Spontaneous spikes were used to trigger PT stimuli at different intervals after the spontaneous spike, and the interval at which the antidromic response was collided was determined. These tests were performed before and after the recording of task-related activity from the PTN; the collision technique also allowed us to confirm that the spontaneous spikes originated from the same neuron as the antidromic spikes.

The ADL of a given PTN is a reflection of axonal conduction velocity, and previous studies have shown that the ADL is also related to cell size (Deschênes et al., 1979; Sakai and Woody, 1988), with the shortest ADL (fastest axons) being recorded from the large pyramidal neurons or Betz cells, which are a characteristic feature of the primary motor cortex.

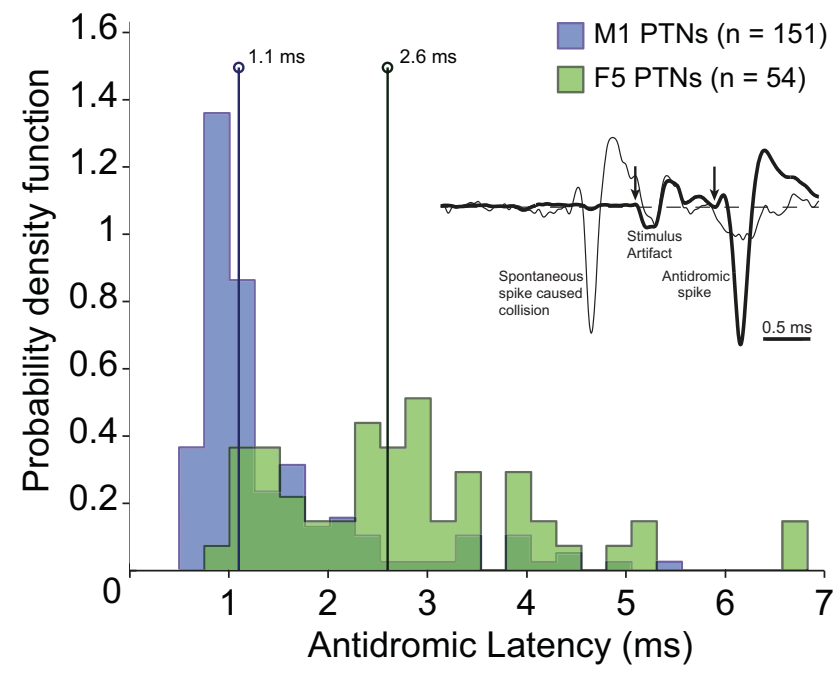

Figure 1. Probability density functions comparing antidromic latencies of identified PTNs. Distributions are shown for M1 (blue) and F5 (green) PTNs. Binwidth $0.25 \mathrm{~ms}$. The two vertical lines correspond to the median antidromic latency for each population of PTNs (1.1 and $2.6 \mathrm{~ms}$ for M1 and F5, respectively). The two median values are significantly different $(p<0.0001$, Wilcoxon rank-sum test). Inset shows the antidromic response of an M1 PTN (heavy trace, average of 40 sweeps). Arrows indicate the onset of the PT stimulus and the onset of the antidromic spike. The antidromic latency of this PTN was $0.9 \mathrm{~ms}$, spike duration was $0.24 \mathrm{~ms}$. The thin trace shows collision of the antidromic spike by a spontaneous spike from this PTN, which occurred just before the PT stimulus.

During the same recording sessions, we regularly encountered spikes with good signal-to-noise ratios that did not respond antidromically to PT stimulation; these were referred to as unidentified neurons (UIDs).

Off-line analysis. PTNs were clustered using modified Wave_clus software (Quian Quiroga et al., 2004; Kraskov et al., 2009). In brief, we used an extended set of features that included not only wavelet coefficients but also the first three principal components. Spike shapes of PTNs obtained after clustering were checked against shapes of spikes that spontaneously collided antidromic spikes during PT stimulation.

Spike duration calculation. We calculated the duration of spontaneous PTN spikes from the negative trough to the succeeding positive peak. This was measured from the averaged spike waveform of the upsampled $(1 \mathrm{MHz})$ spline interpolated individual spikes aligned to the trough (see Fig. $3 A$, examples in inset). The median number of spikes we averaged was 1000. This trough-to-peak measure was chosen for two reasons. First, both trough and peak are easily and reliably detectable. Second, it has been shown that the unfiltered extracellular spike waveform is approximately the derivative of the intracellular action potential, i.e., the trough-to-peak of the extracellular spike is considered to be the equivalent of the spike duration of the intracellular action potential measured at half amplitude (Henze et al., 2000). As a control, we also calculated the spike duration using the first inflection to positive peak measure ("peakto-peak") as used in several previous reports (see Table 2). For this analysis, if there was no clear initial peak, we used the first significant deflection (mean $-2 \mathrm{SD}$ ) as a starting point instead of the initial peak. Identical measurements were made on recordings from UIDs.

\section{Results}

\section{Distribution of antidromic latencies in identified PTNs}

We analyzed data recorded from four monkeys (Table 1). Recordings were made from M1 in all four monkeys (M43: 67 PTNs and 18 UIDs; M44: 31 PTNs and 97 UIDs; monkey L: 35 PTNs, and monkey M 18 PTNs) and from area F5 in M43 (47 PTNs and 55 UIDs) and M44 (7 PTNs and 51 UIDs). In total we recorded from 205 PTNs (151 in M1 and 54 in area F5), and from 221 UIDs (115 in M1 and 106 in area F5).

Figure 1 shows a probability density function for ADLs of M1 (blue) and area F5 (green) PTNs. The M1 ADL distribution was 
Table 1. Database

\begin{tabular}{lllcllr}
\hline Area & Cell type & M43 & M44 & L & M & Total \\
\hline M1 & PTNs & 67 & 31 & 35 & 18 & 151 \\
& UIDs & 18 & 97 & & & 115 \\
F5 & PTNs & 47 & 7 & & & 54 \\
& UIDs & 55 & 51 & & & 106 \\
\hline
\end{tabular}

Numbers of PTNs and UIDs recorded in each of the four monkeys. Only M1 PTNs were analyzed for monkeys L and M.

positively skewed toward short ADLs (range 0.5-5.5 ms, median $1.1 \mathrm{~ms}$ ). However, we also recorded some M1 PTNs with longer ADLs ( $>3.0 \mathrm{~ms}$ ) indicating that we also sampled PTNs belonging to a slower conducting population. In contrast, the distribution of ADLs in area F5 was shifted toward longer ADLs (range 1.0$6.9 \mathrm{~ms}$, median $2.6 \mathrm{~ms}$ ) and is significantly different from the M1 population ( $p<0.0001$, Wilcoxon rank-sum test). Some area F5 PTNs had ADLs as long as 6-7 ms. Assuming a conduction distance of $\sim 50 \mathrm{~mm}$ from cortex to PT stimulating electrode, this equates to an axonal conduction velocity of $<10 \mathrm{~m} / \mathrm{s}$. These PTNs clearly belong to a slower conducting population which are known to far outnumber large ones but are much less studied due to recording bias (Towe and Harding, 1970; Humphrey and Corrie, 1978).

\section{Measurement and distribution of spike duration}

Since the high- and low-pass filter settings can affect the shape of the spike waveform (Quian Quiroga, 2009), we performed additional recordings using wide band filter settings $(10 \mathrm{~Hz}-6 \mathrm{kHz})$ and isolated 25 single units (19 PTNs, 6 UIDs). To estimate the effect of filtering on our measure of spike duration, we digitally filtered the original spike waveforms of the 25 single units (causal, 2-order high-pass Butterworth filter at $300 \mathrm{~Hz}$ ) and plotted the durations of the unfiltered vs filtered spikes (Fig. 2). The data were fitted using a second-order polynomial $\left(R^{2}=0.99\right.$, light blue curve). It is clear from the plot that spike duration was reduced after filtering, and moreover the absolute reduction was much more pronounced for wide spikes than for narrow spikes. The median difference in spike duration for all filtered spikes longer than $0.30 \mathrm{~ms}$ was $0.15 \mathrm{~ms}$, whereas for spikes with durations between 0.20 and $0.30 \mathrm{~ms}$, the reduction was only $0.04 \mathrm{~ms}$. Therefore we concluded that the $300 \mathrm{~Hz}$ high-pass filter used to acquire the main body of data would not have significantly distorted our measurements of spikes with short durations, which were the main focus of this study.

\section{Spike duration of identified PTNs}

Figure 3, $A$ and $B$, shows the distribution of spike durations measured from trough to peak (see Materials and Methods) for PTNs recorded from M1 (blue) and area F5 (green), respectively. The distribution of spike durations in M1 was positively skewed with the majority of spikes having short durations of $0.20-0.25 \mathrm{~ms}$. The shortest values measured were in the range $0.16-0.18 \mathrm{~ms}$. The distribution in area F5 was rather different, with one group of PTNs having short spike durations (0.15-0.30 $\mathrm{ms})$ and the other longer durations $(0.35-0.50 \mathrm{~ms})$. While the range of spike durations in M1 (0.16-0.71 ms; Fig. 3) was similar to that found in area F5 $(0.15-0.71 \mathrm{~ms})$, the median spike duration of PTNs in M1 (0.26 ms) was significantly shorter compared with PTNs in area F5 $(0.43 \mathrm{~ms})(p<0.001$, Wilcoxon rank-sum test). The median value for all 205 PTNs was $0.29 \mathrm{~ms}$.

\section{PTNs versus unidentified neurons}

We next compared the spike duration of the PTN population with a population of unidentified neurons (UIDs; see Materials

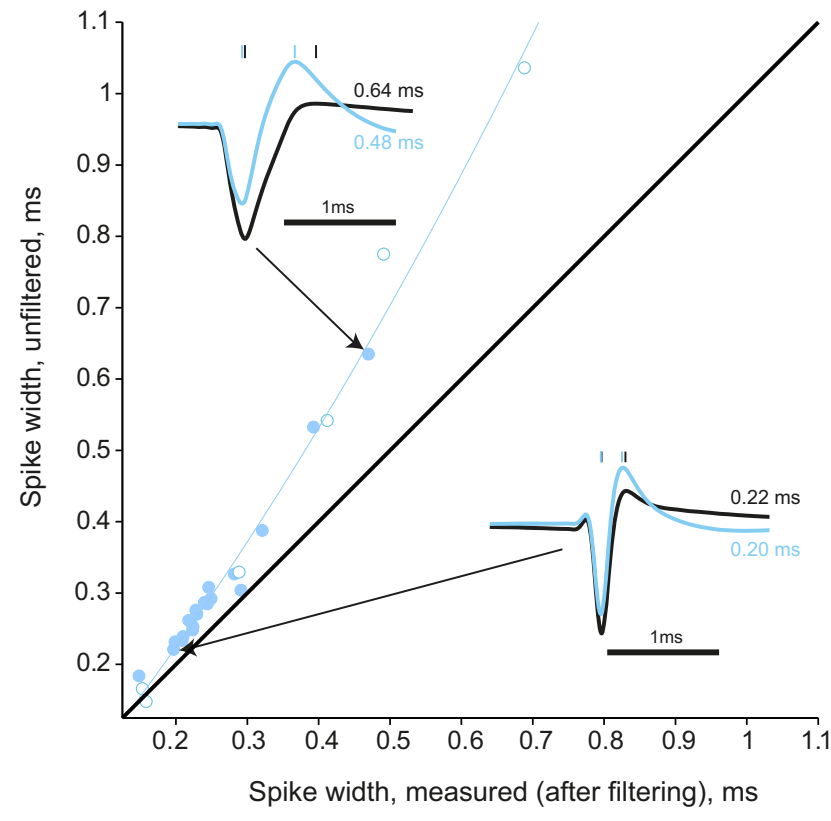

Figure 2. Comparison of spike duration for recordings in filtered versus unfiltered conditions. The data points (light blue circles) have been approximated with a second-order polynomial ( $R^{2}=0.99$, light blue curve). Open circles correspond to UIDs and filled ones to PTNs. For filtered recordings we used a second-order causal high-pass Butterworth filter with a cutoff frequency of $300 \mathrm{~Hz}$ (the same filter as used in all the recordings reported here). Thick black line is the line of unity. The two insets show samples of unfiltered spike waveforms (black traces) from two PTNs, one with a narrow spike duration $(0.22 \mathrm{~ms})$ and one with a relatively wide spike $(0.64 \mathrm{~ms})$ and their filtered versions (light blue traces). All waveforms are averages of 1000 spikes. The duration of the filtered narrow spike decreased by $0.02 \mathrm{~ms}$ ( $11 \%$ reduction) whereas the filtered wide spike was reduced by $0.16 \mathrm{~ms}$ ( $26 \%$ reduction).

and Methods), many of which were recorded simultaneously from other microelectrodes whose tips were located not $>1 \mathrm{~mm}$ away from the sampled PTNs. The combination of these two distributions should closely resemble a typical population of neurons recorded without PT identification being applied, and potentially contain some interneurons. Since PTN spike durations were found to be different in area M1 and area F5, we compared PTNs and UIDs within the same area. Figure $4 A$ shows the probability density function of spike duration distribution of PTNs $(n=151)$ vs UIDs $(n=115)$ in M1 recordings. Importantly, these distributions are not statistically different $(p>0.8$, Wilcoxon rank-sum test) with very similar median values $(0.26$ and $0.27 \mathrm{~ms}$, PTNs and UIDs, respectively) and range $(0.16-0.71 \mathrm{~ms}$ for PTNs vs $0.13-0.70 \mathrm{~ms}$ for UIDs). Thus, although the distribution suggests that there was a larger population of UIDs with very short spike durations, there was almost complete overlap in terms of actual spike duration. We also compared area F5 PTNs $(n=54)$ with UIDs $(n=106)$ (Fig. $4 B)$. The distributions of these two populations were also overlapping (0.15-0.71 ms for PTNs vs $0.14-0.80 \mathrm{~ms}$ for UIDs). Although the median value for the PTNs $(0.43 \mathrm{~ms})$ was slightly longer than for the UIDs $(0.35$ $\mathrm{ms})$ they were not significantly different $(p>0.2$, Wilcoxon rank-sum test).

\section{Positive correlation of PTN antidromic latency with spike duration}

We subsequently performed a linear regression analysis between PTN spike duration and antidromic latency (Fig. 5), since the latter is known to reflect PTN soma size (Deschênes et al., 1979; Sakai and Woody, 1988). We found a strong significant positive 

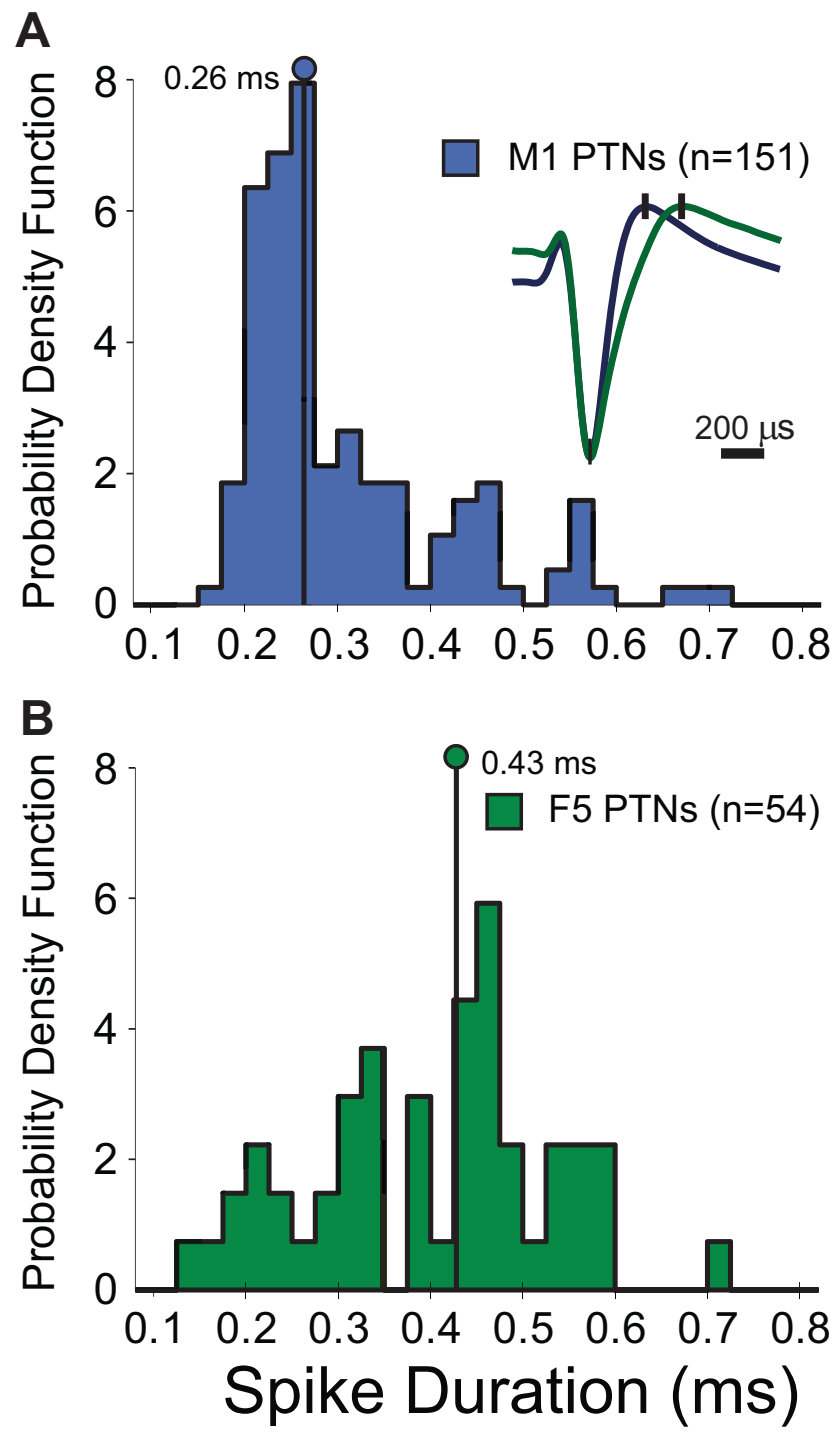

Figure 3. Spike durations of PTNs in M1 and F5. $A$, Probability density function of spike durations of identified PTNs in M1 (blue). Binwidth $0.025 \mathrm{~ms}$. Vertical line corresponds to the median spike duration ( $0.26 \mathrm{~ms}$ ). $\boldsymbol{B}$, Probability density function of spike durations of identified PTNs in F5 (green). The median spike duration of M1 PTNs ( $0.26 \mathrm{~ms}$ ) was significantly shorter than that for PTNs in F5 ( $0.43 \mathrm{~ms}$ ) ( $p<0.001$, Wilcoxon rank-sum test). Inset shows splined averaged waveforms for two PTNs from M1 (blue, $n=32,943$ spikes) and F5 (green, $n=$ 1070). These waveforms have spike durations closest to the medians of their respective populations indicated in the main figure.

correlation for both M1 and area F5 PTN populations $\left(\mathrm{M} 1, R^{2}=\right.$ $\left.0.40 ; \mathrm{F} 5, R^{2}=0.57, p<0.001\right)$. Figure 5 shows the scatter plot and regression line for all the PTNs in the sample $(n=205)$. M1 and area F5 populations shared the same linear relationship between antidromic latency and spike duration. A linear regression for each individual monkey and on the combined data were also highly significant $\left(R^{2}=0.51, p<0.001\right)$.

The relationship implies that the cells with the shortest ADLs and thus the fastest axons and probably largest somas exhibit the shortest spike durations, as assessed by the trough-to-peak measure. We also found a significant correlation between ADLs and spike durations measured from peak to peak (see Materials and Methods, $R^{2}=0.41, p<0.001$ ), which is unsurprising given that the two measures of spike duration are highly correlated (Fig. 6; $\left.R^{2}=0.80, p<0.0001\right)$. The slope of linear regression is 1.2 , $[1.12-1.29,95 \% \mathrm{CI}]$, with an intercept of $106 \mu \mathrm{s}$; we used this to
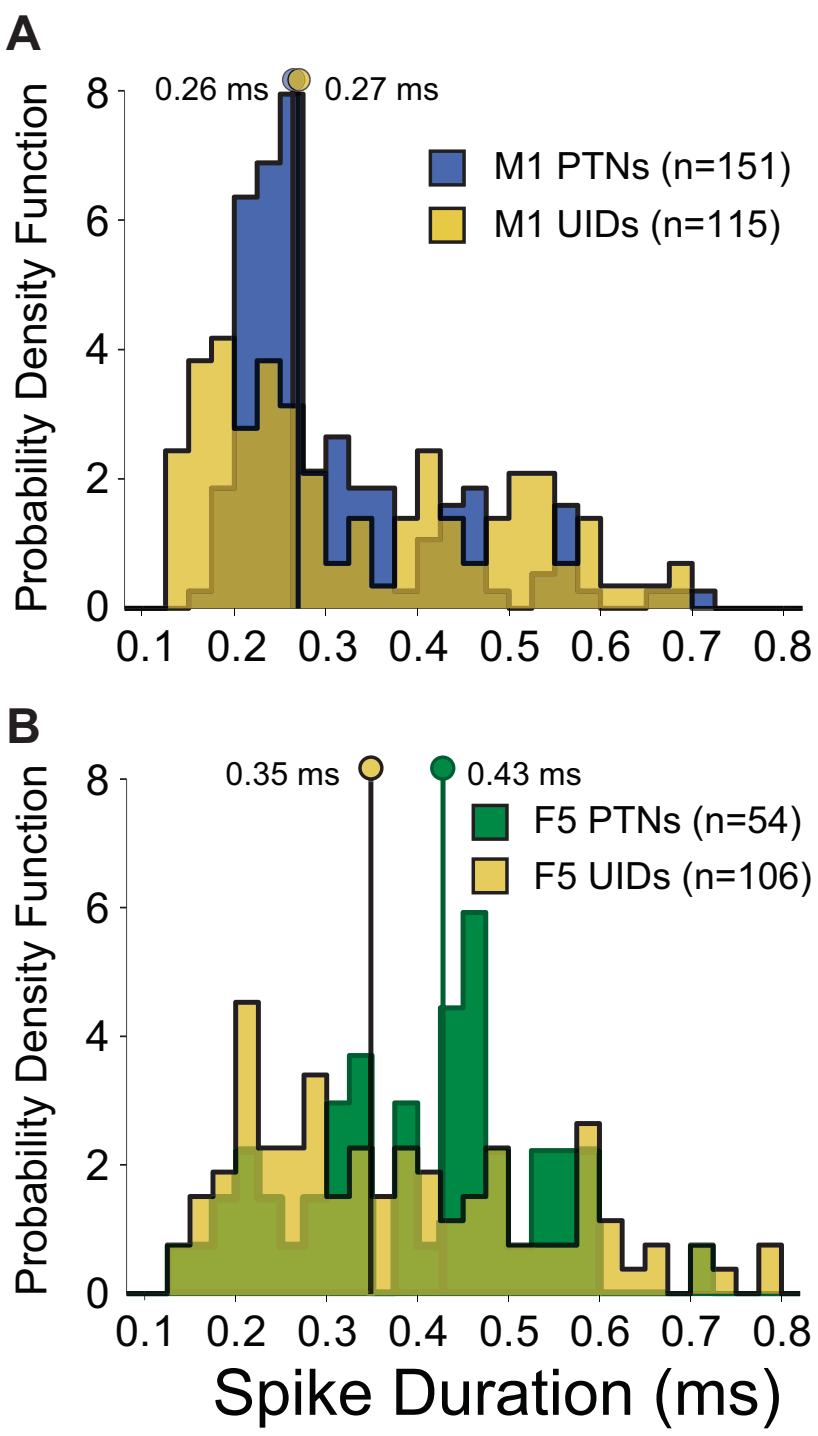

Figure 4. Spike durations of PTNs and unidentified neurons. $A$, Probability density function comparing spike durations of identified PTNs in M1 (blue) and M1 UIDs (yellow). Binwidth 0.025 ms. The two vertical lines correspond to the median spike duration for each population. The median spike duration of PTNs in M1 $(0.26 \mathrm{~ms})$ was not significantly different from that of UIDs in the same area $(0.27 \mathrm{~ms})(p>0.8$, Wilcoxon rank-sum test). Note that the UID population appears bimodal with a trough in the distribution at $\sim 0.4 \mathrm{~ms}$ and there is extensive overlap between the pyramidal and UID populations. $\boldsymbol{B}$, Probability density function comparing spike durations of identified PTNs in F5 (green) and F5 UIDs (yellow). The two vertical lines correspond to the median spike duration for each population. The median spike duration of PTNs in F5 (0.43 ms) was again not significantly different from that of UIDs in the same area $(0.35 \mathrm{~ms})(p>0.2$, Wilcoxon rank-sum test). Note that there is considerable overlap between the two populations.

estimate the average trough-to-peak spike duration from peakto-peak analyses reported in the literature (Table 2). Among the fast PTNs in M1 (ADL $<2.0 \mathrm{~ms}$ ), we found no significant correlation between the amplitude of recorded spikes and spike duration $(p=0.29)$, suggesting that proximity of the electrode to a PTN did not influence the duration of the recorded spike.

\section{Discussion}

Our results demonstrate that physiologically identified PTNs, recorded in the motor cortex of the awake monkey, exhibit a wide range of spike durations. PTNs with short antidromic latencies generated the narrowest spikes, in the order of $0.15-0.17 \mathrm{~ms}$, while those with longer latencies have much broader spikes, up to 


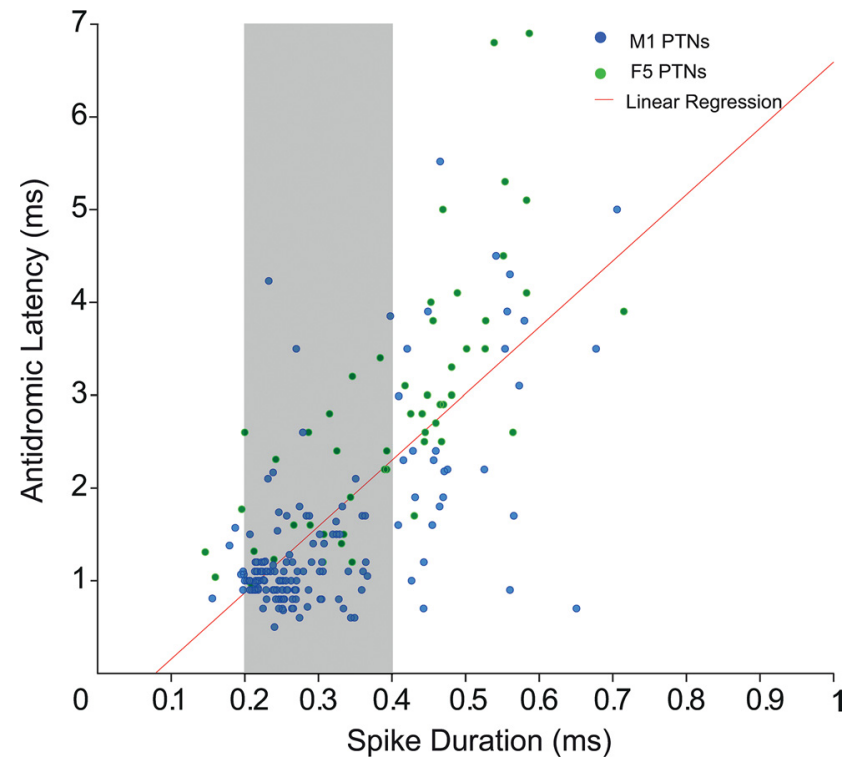

Figure 5. Positive correlation between PTN axon conduction velocity and spike duration. Scatter plot showing the positive correlation between antidromic latency (a surrogate for axonal conduction velocity and cell size) and spike duration for identified PTNs in areas M1 (blue) and F5 (green). The data have been fitted with a linear regression line shown in red. The correlation was highly significant $\left(R^{2}=0.51, p<0.001\right)$. The light gray box indicates the range of spike durations $(0.2-0.4 \mathrm{~ms})$ that have been cited in the literature as a means of discriminating interneurons from pyramidal cells on the basis of spike duration (Table 2; see Discussion).

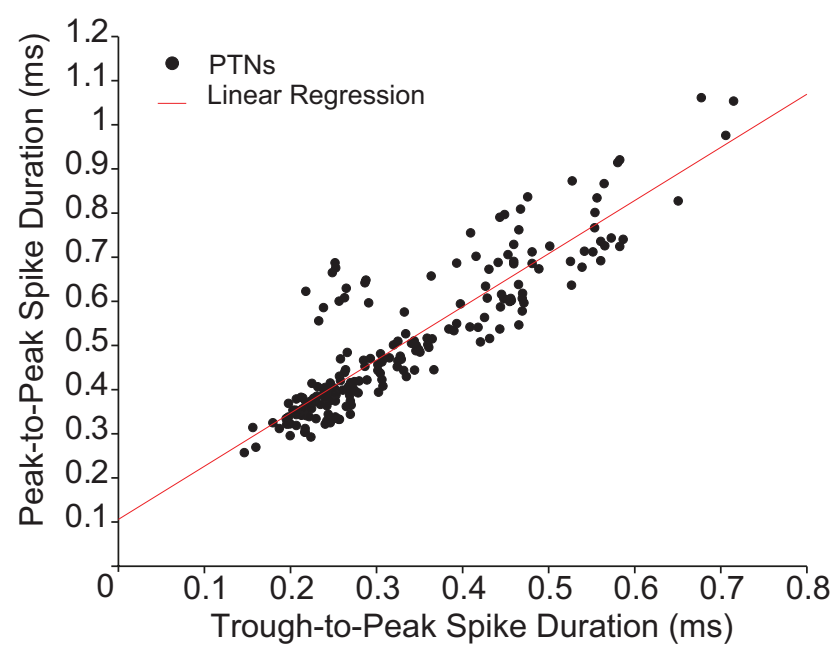

Figure 6. Relationship between peak-to-trough and peak-to-peak measures of spike duration. Scatter plot showing the relationship between spike duration as measured from the first negative trough to the subsequent peak of the extracellular waveform (trough-to-peak, as used in previous figures) and as measured from the first positive peak to the subsequent peak (peakto-peak measure). Data from all identified PTNs in areas M1 and F5 (filled black circles). There was a significant correlation between the two measures of spike duration $\left(R^{2}=0.80, p<\right.$ 0.0001). Note that the slope of the regression line (1.2, shown in red) and the intercept (106 $\mu \mathrm{s})$ can be used to compare our measure of spike duration with others given in the literature (Table 2).

$0.70 \mathrm{~ms}$. PTNs with very narrow or thin spikes were not confined to M1 but some were also found in a "secondary" motor region, area F5 of the ventral premotor cortex (Fig. 3). There was a significant positive correlation between $\mathrm{ADL}$ and spike duration for the whole sample of PTNs (Fig. 5), but also for the two subpopulations of M1 and F5 PTNs. To our knowledge, this is the first study in the awake monkey highlighting the fact that pyramidal neurons can exhibit thin spikes.

\section{Previous studies comparing spike durations of neocortical neurons}

Spike duration has been suggested as one means of distinguishing putative neocortical interneurons from pyramidal neurons. Table 2 summarizes studies in which spike duration has been reported, including the type and conditions of recording and the spike features measured. Some of the studies were performed in awake macaques, and involved extracellular recordings from unidentified neurons in a variety of cortical motor (M1, dorsal premotor cortex), visual (V1, V4) and prefrontal areas. All of them used the "trough-to-peak" measure of spike duration and, based on data from other species and cortical areas (Table 2; see Discussion), all concluded that it was possible to distinguish interneurons by their short spike duration, although the boundary value varies from $0.19 \mathrm{~ms}$ (Kaufman et al., 2010) to $0.40 \mathrm{~ms}$ (Gur et al., 1999).

\section{Spike durations in identified pyramidal neurons}

The early intracellular study by Calvin and Sypert (1976) of PTNs in the motor cortex of the anesthetized cat showed a clear positive correlation between spike duration and antidromic latency (their Fig. $1 B$ ). Similarly, fast PTNs recorded intracellularly in motor cortex of awake cats had brief spikes (mean $0.41 \mathrm{~ms}$, range 0.30 $0.80 \mathrm{~ms}$, measured at half amplitude) (Baranyi et al., 1993). Chen et al. (1996a,b) made intracellular recordings from cat motor cortex slices, and reported "narrow spiking" in neurons revealed by subsequent intracellular staining to be large lamina V pyramidal cells. It is remarkable that these studies in the cat are not cited by those working with the awake monkey.

In the current study, the median spike duration for identified PTNs in M1 $(0.26 \mathrm{~ms})$ and in area F5 $(0.43 \mathrm{~ms})$ are considerably briefer than the estimated mean spike duration for the population of "putative" pyramidal cells in all the studies listed in Table $2(\sim 0.55 \mathrm{~ms})$, and the M1 value is shorter than any of the listed macaque studies. More importantly, the mean spike duration of putative interneurons listed in Table 2 is longer than the median spike duration of identified M1 PTNs in our study. There clearly exists a population of PTNs with thin spikes having durations smaller than the boundary value between putative interneurons and pyramidal cells reported in any of the cited studies (Fig. 5). That is, without PT identification, these PTNs would have been erroneously classified as interneurons.

\section{Pyramidal neurons in M1 versus other cortical areas: the significance of cell size}

It could be argued that M1, with its characteristically large pyramidal neurons, is a special case and that in recordings from other cortical areas the interneuron-pyramidal distinction based on spike duration could still be applied. Corticospinal neurons in areas such as ventral premotor cortex and SMA are smaller than those in M1 (Murray and Coulter, 1981) and have slower conduction velocities (Macpherson et al., 1982; Maier et al., 2002; Kraskov et al., 2009). Isolated examples of recordings in this study from PTNs in somatosensory cortex confirmed this impression: an area $3 \mathrm{a}$ PTN had a long ADL of $3.7 \mathrm{~ms}$ and spike duration of $0.36 \mathrm{~ms}$, and an area $2 \mathrm{PTN}$ had an ADL of $4.8 \mathrm{~ms}$ and spike duration of $0.50 \mathrm{~ms}$.

However, our results suggest considerable caution even for recordings made beyond M1. Although F5 PTNs had significantly longer ADLs (Fig. 1), there is considerable scatter in the 
Table 2. Literature review

\begin{tabular}{|c|c|c|c|c|c|}
\hline Paper & Paper criterion & Putative interneurons/FS (ms) & Putative pyramidal/RS (ms) & Animal, brain area & Condition \\
\hline Mountcastle et al., 1969 & - & $0.1-0.3$ & $0.3-0.5$ & Macaque, S1 & Awake \\
\hline Gur et al., 1999 & $\mathrm{~T} 2 \mathrm{P}$ & $<0.4$ & $>0.4$ & Macaque, $\mathrm{V} 1$ & Awake \\
\hline Constantinidis and Goldman-Rakic, 2002 & P2P (Inv) & 0.47 & 0.64 & Macaque, DLPFC & Awake \\
\hline Cohen et al., 2009 & $\mathrm{~T} 2 \mathrm{P}$ & 0.22 & N/A & Macaque, FEF & Awake \\
\hline Merchant et al., 2008 & P2P (Inv) & 0.42 & 0.80 & Macaque, M1 & Awake \\
\hline Diester and Nieder, 2008 & $\mathrm{~T} 2 \mathrm{P}$ & $<0.28$ & $>0.28$ & Macaque, PFC & Awake \\
\hline Song and McPeek, 2010 & $\mathrm{~T} 2 \mathrm{P}$ & $0.1-0.3$ & $0.3-0.5$ & Macaque, PMd & Awake \\
\hline Krimer et al., 2005 & IHA & $<0.4$ & $>0.4$ & Macaque, DLPFC & In vitro \\
\hline Zaitsev et al., 2009 & IHA & $0.32-0.74$ & $N / A$ & Macaque, DLPFC & In vitro \\
\hline McCormick et al., 1985 & IHA & 0.32 & 0.8 & Guinea Pig, CC & In vitro \\
\hline Swadlow, 1988 & P2P & 0.47 & 0.98 & Rabbit, V1 & Awake \\
\hline Swadlow, 1989 & P2P & 0.43 & 0.98 & Rabbit, S1 & Awake \\
\hline
\end{tabular}

The mean value for the spike duration of putative pyramidal cells is $\sim 0.55 \mathrm{~ms}$, which has been calculated either using the numbers reported in the paper or estimated from the figures. FS, Fast spiking; RS, regular spiking; T2P, trough to peak; P2P, peak to peak; Inv, inverted spike; IHA, intracellular spike duration at half-amplitude; S1, V1, M1, primary visual, somatosensory, motor cortex; CC, cingulate cortex; FEF, frontal eye fields. PMd, dorsal premotor cortex; DLPFC, dorsolateral prefrontal cortex.

duration-ADL regression (Fig. 5), and we encountered a considerable proportion of area F5 PTNs with short-duration (0.15$0.30 \mathrm{~ms}$ ) spikes (Fig. 3; green dots in Fig. $5 ; 12$ of $54=23 \%$ ). The single population of PTNs in area F5 clearly comprised those with narrow vs broad spikes (Figs. 3, 4), and so a bimodal distribution in spike duration per se cannot be taken as evidence of recordings from different cell types.

It could also be argued that corticospinal neurons represent a special case, with large cell bodies and fast-conducting axons very different from other types of pyramidal neuron, such as corticostriatal neurons (Turner and DeLong, 2000), callosal neurons (Soteropoulos and Baker, 2007) and corticocortical neurons (Godschalk et al., 1984; Kraskov et al., 2011) which have shorter axons, lower conduction velocities $(<20 \mathrm{~m} / \mathrm{s})$ and broad spikes (Soteropoulos and Baker, 2007). However, there are numerous corticofugal neurons making longer projections to the brainstem and pons, which might be anticipated to have large axons (Tomasch, 1969; Glickstein et al., 1985; Turner and DeLong, 2000).

\section{Comparison of PTNs with UIDs}

Figure 4 shows a substantial overlap between the spike durations of PTNs and UIDs, in both area F5 and M1. Some UIDs in both areas also had brief spikes. One interpretation is that the UID sample contained a significant proportion of interneurons (cf. Merchant et al., 2008), in which case it emphasizes the almost complete overlap between the spike durations of these interneurons and identified PTNs. However, the likelihood of recordings from interneurons should be reduced both by their small size and their relatively small contribution to the total population of cortical neurons (Sloper et al., 1979). Thus another interpretation is that the UID recordings were from other pyramidal neurons, whose axons do not travel in the pyramidal tract, suggesting that the PTNs we have sampled were representative of the pyramidal population in these cortical areas.

One of the few studies in which records were made from identified neurons in monkey prefrontal cortex slices (Krimer et al., 2005) pointed out that there may be overlap between the spike durations of regularly spiking pyramidal cells and at least one type of interneuron. Morphologically identified cortical interneurons can show a wide range of spike durations (0.32-0.74 ms) (Zaitsev et al., 2009).

\section{Comparative biology of pyramidal neurons}

Our data suggest that macaque PTNs can have briefer spikes than those found in the cat (Sakai and Woody, 1988; Baranyi et al., 1993). This is probably is partly explained by the presence of a larger, faster conducting subpopulation of PTNs in the monkey not found in the cat (Evarts, 1965; Humphrey and Corrie, 1978; Nudo et al., 1995). Importantly, the broad spikes recorded in vivo and in vitro from pyramidal cells in rodent, guinea pig and rabbit cortex (Table 2) may reflect the smaller size of pyramidal neurons in these species (Donoghue and Kitai, 1981; Landry et al., 1984; Nudo et al., 1995). For example, in the rat and rabbit the largest pyramidal neurons have conduction velocities of $<20 \mathrm{~m} / \mathrm{s}$ (Mediratta and Nicoll, 1983) and relatively small somata (Landry et al., 1984; Nudo et al., 1995). Spike duration criteria developed in one animal model (Barthó et al., 2004) cannot necessarily be applied to other species.

\section{What is the underlying mechanism of the fast spike duration in large pyramidal neurons?}

The trough to peak of the extracellular spike waveform encompasses the repolarization phase of the membrane potential (Henze et al., 2000). Differences in spike duration between interneurons and pyramidal cells in rodents are partly due to different levels of expression of $\mathrm{Na}^{+}$and $\mathrm{K}^{+}$channels (Martina and Jonas, 1997; Martina et al., 1998; Erisir et al., 1999). Fast-spiking properties reflect the presence of Kv3 and Kv1 channels which allow fast repolarization. Kv3.1b mRNA and protein are associated with fast spiking interneurons in rodents (Kawaguchi and Kubota, 1997; Härtig et al., 1999), but, in keeping with our results, these markers are also expressed by large layer $\mathrm{V}$ pyramidal cells in macaque motor cortex (Ichinohe et al., 2004). We speculate that large and fast PTNs might express more Kv3.1b allowing shorter spike durations and the higher firing rates of fast PTNs first reported by Evarts (1965).

\section{Conclusion}

In summary, our study confirms for the awake monkey previous findings in the cat motor cortex: thin spikes can originate from pyramidal neurons. It extends this observation to PTNs recorded in a secondary motor area. We conclude that spike duration alone may not provide a reliable indication of cell type, at least in areas 
of primate cortex containing PTNs, but more likely reflects discharge properties shared between cortical interneurons and some pyramidal neurons.

\section{References}

Baker SN, Philbin N, Spinks R, Pinches EM, Wolpert DM, MacManus DG, Pauluis Q, Lemon RN (1999) Multiple single unit recording in the cortex of monkeys using independently moveable microelectrodes. J Neurosci Methods 94:5-17.

Baranyi A, Szente MB, Woody CD (1993) Electrophysiological characterization of different types of neurons recorded in vivo in the motor cortex of the cat. II. Membrane parameters, action potentials, current-induced voltage responses and electrotonic structures. J Neurophysiol 69:1865-1879.

Barthó P, Hirase H, Monconduit L, Zugaro M, Harris KD, Buzsáki G (2004) Characterization of neocortical principal cells and interneurons by network interactions and extracellular features. J Neurophysiol 92:600-608.

Calvin WH, Sypert GW (1976) Fast and slow pyramidal tract neurons: an intracellular analysis of their contrasting repetitive firing properties in the cat. J Neurophysiol 39:420-434.

Chen W, Zhang JJ, Hu GY, Wu CP (1996a) Electrophysiological and morphological properties of pyramidal and nonpyramidal neurons in the cat motor cortex in vitro. Neuroscience 73:39-55.

Chen W, Zhang JJ, Hu GY, Wu CP (1996b) Different mechanisms underlying the repolarization of narrow and wide action potentials in pyramidal cells and interneurons of cat motor cortex. Neuroscience 73:57-68.

Cohen JY, Pouget P, Heitz RP, Woodman GF, Schall JD (2009) Biophysical support for functionally distinct cell types in the frontal eye field. J Neurophysiol 101:912-916.

Connors BW, Gutnick MJ, Prince DA (1982) Electrophysiological properties of neocortical neurons in vitro. J Neurophysiol 48:1302-1320.

Constantinidis C, Goldman-Rakic PS (2002) Correlated discharges among putative pyramidal neurons and interneurons in the primate prefrontal cortex. J Neurophysiol 88:3487-3497.

Contreras D (2004) Electrophysiological classes of neocortical neurons. Neural Netw 17:633-646.

Deschênes M, Labelle A, Landry P (1979) Morphological characterization of slow and fast pyramidal tract cells in the cat. Brain Res 178:251-274.

Diester I, Nieder A (2008) Complementary contributions of prefrontal neuron classes in abstract numerical categorization. J Neurosci 28:7737-7747.

Donoghue JP, Kitai ST (1981) A collateral pathway to the neostriatum from corticofugal neurons of the rat sensory-motor cortex: an intracellular HRP study. J Comp Neurol 201:1-13.

Dum RP, Strick PL (1991) The origin of corticospinal projections from the premotor areas in the frontal-lobe. J Neurosci 11:667-689.

Erisir A, Lau D, Rudy B, Leonard CS (1999) Function of specific K(+) channels in sustained high-frequency firing of fast-spiking neocortical interneurons. J Neurophysiol 82:2476-2489.

Evarts EV (1964) Temporal patterns of discharge of pyramidal tract neurons during sleep and waking in the monkey. J Neurophysiol 27:152-171.

Evarts EV (1965) Relation of discharge frequency to conduction velocity in pyramidal tract neurons. J Neurophysiol 28:216-228.

Glickstein M, May JG 3rd, Mercier BE (1985) Corticopontine projection in the macaque: the distribution of labelled cortical cells after large injections of horseradish peroxidase in the pontine nuclei. J Comp Neurol 235:343-359.

Godschalk M, Lemon RN, Kuypers HG, Ronday HK (1984) Cortical afferents and efferents of monkey postarcuate area-an anatomical and electrophysiological study. Exp Brain Res 56:410-424.

Gold C, Henze DA, Koch C, Buzsáki G (2006) On the origin of the extracellular action potential waveform: a modeling study. J Neurophysiol 95:3113-3128.

Gur M, Beylin A, Snodderly DM (1999) Physiological properties of macaque V1 neurons are correlated with extracellular spike amplitude, duration, and polarity. J Neurophysiol 82:1451-1464.

Härtig W, Derouiche A, Welt K, Brauer K, Grosche J, Mäder M, Reichenbach A, Brückner G (1999) Cortical neurons immunoreactive for the potassium channel Kv3.1b subunit are predominantly surrounded by perineuronal nets presumed as a buffering system for cations. Brain Res 842:15-29.

Henze DA, Borhegyi Z, Csicsvari J, Mamiya A, Harris KD, Buzsáki G (2000)
Intracellular features predicted by extracellular recordings in the hippocampus in vivo. J Neurophysiol 84:390-400.

Humphrey DR, Corrie WS (1978) Properties of pyramidal tract neuron system within a functionally defined subregion of primate motor cortex. J Neurophysiol 41:216-243.

Ichinohe N, Watakabe A, Miyashita T, Yamamori T, Hashikawa T, Rockland KS (2004) A voltage-gated potassium channel, Kv3.1b, is expressed by a subpopulation of large pyramidal neurons in layer 5 of the macaque monkey cortex. Neuroscience 129:179-185.

Kaufman MT, Churchland MM, Santhanam G, Yu BM, Afshar A, Ryu SI, Shenoy KV (2010) Roles of monkey premotor neuron classes in movement preparation and execution. J Neurophysiol 104:799-810.

Kawaguchi Y, Kubota Y (1997) GABAergic cell subtypes and their synaptic connections in rat frontal cortex. Cereb Cortex 7:476-486.

Kraskov A, Dancause N, Quallo MM, Shepherd S, Lemon RN (2009) Corticospinal neurons in macaque ventral premotor cortex with mirror properties: a potential mechanism for action suppression? Neuron 64:922-930.

Kraskov A, Prabhu G, Quallo MM, Lemon RN, Brochier T (2011) Ventral premotor-motor cortex interactions in the macaque monkey during grasp: response of single neurons to intracortical microstimulation. J Neurosci 31:8812-8821.

Krimer LS, Zaitsev AV, Czanner G, Kröner S, González-Burgos G, Povysheva NV, Iyengar S, Barrionuevo G, Lewis DA (2005) Cluster analysis-based physiological classification and morphological properties of inhibitory neurons in layers $2-3$ of monkey dorsolateral prefrontal cortex. J Neurophysiol 94:3009-3022.

Landry P, Wilson CJ, Kitai ST (1984) Morphological and electrophysiological characteristics of pyramidal tract neurons in the rat. Exp Brain Res $57: 177-190$

Lemon R (1984) Methods for neuronal recording in conscious animals. Chichester, UK: Wiley.

Lemon RN (2008) Descending pathways in motor control. Annu Rev Neurosci 31:195-218.

Macpherson J, Wiesendanger M, Marangoz C, Miles TS (1982) Corticospinal neurones of the supplementary motor area of monkeys. A single unit study. Exp Brain Res 48:81-88.

Maier MA, Armand J, Kirkwood PA, Yang HW, Davis JN, Lemon RN (2002) Differences in the corticospinal projection from primary motor cortex and supplementary motor area to macaque upper limb motoneurons: an anatomical and electrophysiological study. Cereb Cortex 12:281-296.

Martina M, Jonas P (1997) Functional differences in Na+ channel gating between fast-spiking interneurones and principal neurones of rat hippocampus. J Physiol 505:593-603.

Martina M, Schultz JH, Ehmke H, Monyer H, Jonas P (1998) Functional and molecular differences between voltage-gated $\mathrm{K}+$ channels of fastspiking interneurons and pyramidal neurons of rat hippocampus. J Neurosci 18:8111-8125.

McCormick DA, Connors BW, Lighthall JW, Prince DA (1985) Comparative electrophysiology of pyramidal and sparsely spiny stellate neurons of the neocortex. J Neurophysiol 54:782-806.

Mediratta NK, Nicoll JA (1983) Conduction velocities of corticospinal axons in the rat studied by recording cortical antidromic responses. J Physiol 336:545-561.

Merchant H, Naselaris T, Georgopoulos AP (2008) Dynamic sculpting of directional tuning in the primate motor cortex during three-dimensional reaching. J Neurosci 28:9164-9172.

Mitchell JF, Sundberg KA, Reynolds JH (2007) Differential attentiondependent response modulation across cell classes in macaque visual area V4. Neuron 55:131-141.

Mountcastle VB, Talbot WH, Sakata H, Hyvärinen J (1969) Cortical neuronal mechanisms in flutter-vibration studied in unanesthetized monkeys. Neuronal periodicity and frequency discrimination. J Neurophysiol 32:452-484.

Murray EA, Coulter JD (1981) Organization of corticospinal neurons in the monkey. J Comp Neurol 195:339-365.

Nudo RJ, Sutherland DP, Masterton RB (1995) Variation and evolution of mammalian corticospinal somata with special reference to primates. J Comp Neurol 358:181-205.

Olivier E, Baker SN, Nakajima K, Brochier T, Lemon RN (2001) Investiga- 
tion into non-monosynaptic corticospinal excitation of macaque upper limb single motor units. J Neurophysiol 86:1573-1586.

Quian Quiroga R (2009) What is the real shape of extracellular spikes? J Neurosci Methods 177:194-198.

Quiroga RQ, Nadasdy Z, Ben-Shaul Y (2004) Unsupervised spike detection and sorting with wavelets and superparamagnetic clustering. Neural Comput 16:1661-1687.

Sakai H, Woody CD (1988) Relationships between axonal diameter, soma size, and axonal conduction velocity of HRP-filled, pyramidal tract cells of awake cats. Brain Res 460:1-7.

Sloper JJ, Hiorns RW, Powell TP (1979) A qualitative and quantitative electron microscopic study of the neurons in the primate motor and somatic sensory cortices. Philos Trans R Soc Lond B Biol Sci 285:141-171.

Song JH, McPeek RM (2010) Roles of narrow- and broad-spiking dorsal premotor area neurons in reach target selection and movement production. J Neurophysiol 103:2124-2138.

Soteropoulos DS, Baker SN (2007) Different contributions of the corpus callosum and cerebellum to motor coordination in monkey. J Neurophysiol 98:2962-2973.
Swadlow HA (1988) Efferent neurons and suspected interneurons in binocular visual cortex of the awake rabbit: receptive fields and binocular properties. J Neurophysiol 59:1162-1187.

Swadlow HA (1989) Efferent neurons and suspected interneurons in S-1 vibrissa cortex of the awake rabbit: receptive fields and axonal properties. J Neurophysiol 62:288-308.

Tomasch J (1969) The numerical capacity of the human corticopontocerebellar system. Brain Res 13:476-484.

Towe AL, Harding GW (1970) Extracellular microelectrode sampling bias. Exp Neurol 29:366-381.

Turner RS, DeLong MR (2000) Corticostriatal activity in primary motor cortex of the macaque. J Neurosci 20:7096-7108.

Witham CL, Baker SN (2007) Network oscillations and intrinsic spiking rhythmicity do not covary in monkey sensorimotor areas. J Physiol 580:801-814.

Zaitsev AV, Povysheva NV, Gonzalez-Burgos G, Rotaru D, Fish KN, Krimer LS, Lewis DA (2009) Interneuron diversity in layers $2-3$ of monkey prefrontal cortex. Cereb Cortex 19:1597-1615. 